\title{
Alignment and divergence in European and North American aortic stenosis guidelines
}

\author{
Catherine M. Otto*, MD \\ Division of Cardiology, Department of Medicine, University of Washington School of Medicine, Seattle, WA, USA
}

\begin{abstract}
Major advances in treatment options for aortic valve stenosis (AS) over the past decade have left clinicians with little guidance about optimal timing and types of interventions for patients with severe AS. This situation has been remedied (at least for now) by the recent online publication of the 2021 ESC/EACTS Guidelines for Management of Valvular Heart Disease also published in this issue $^{1}$, and the 2020 ACC/AHA Guideline for Management of Patients with Valvular Heart Disease ${ }^{2}$. Both these professional society guidelines rank the strength of each recommendation on a scale from "recommended" (Class I), to "reasonable" (Class IIa) or "may be considered" (Class IIb), to "not recommended" (Class III). Both documents indicate that recommendations are based on a critical evaluation of published data, with quality of the supporting evidence rated as high, medium or low depending on whether data are derived from multiple randomised clinical trials, large non-randomised studies or less rigorous data sources. However, although these evidence-based guidelines start with the same evidence, the resultant recommendations are not identical.
\end{abstract}

\section{Article, see page 1126}

\section{Timing of intervention}

\section{SYMPTOMATIC PATIENTS WITH SEVERE VALVULAR AS}

Reassuringly, the ESC/EACTS and ACC/AHA recommendations on the timing of intervention for adults with severe valvular AS parallel each other closely, with subtle, but no major conceptual discrepancies. Aortic valve replacement (AVR) is recommended for symptomatic patients with severe AS, defined either as highgradient (aortic velocity $\geq 4.0 \mathrm{~m} / \mathrm{s}$, mean gradient $\geq 40 \mathrm{mmHg}$ ) or low-gradient with low-flow due to a low left ventricular (LV) ejection fraction (EF) (mean gradient $<40 \mathrm{mmHg}$, stroke volume index $\leq 35 \mathrm{ml} / \mathrm{m}^{2}$ and $\mathrm{EF}<50 \%$ ). There are minor differences in that the ESC guidelines also require a valve area $\leq 1.0 \mathrm{~cm}^{2}$ for diagnosis of high-gradient severe $\mathrm{AS}$, whereas the $\mathrm{ACC} / \mathrm{AHA}$ guidelines require only a high velocity/gradient (regardless of valve area) when leaflets are calcified with reduced systolic opening. Importantly, in both guidelines, patients with severe AS are considered symptomatic whether symptoms are reported clinically or provoked by exercise testing. Similarly, a decline in blood pressure on exercise testing is considered a sign of imminent symptom onset warranting AVR.

\section{SYMPTOMATIC LOW-FLOW LOW-GRADIENT AS}

In patients with low-flow, low-gradient severe AS and a reduced $\mathrm{EF}$, both guidelines recommend a low-dose dobutamine stress echo to ensure that AS is severe, based on the valve area remaining $\leq 1.0 \mathrm{~cm}^{2}$. While ESC guidelines recommend AVR when contractile flow reserve (defined as a $\geq 20 \%$ in stroke volume) or severe leaflet calcification is present, ACC/AHA guidelines instead require only an aortic velocity over $4 \mathrm{~m} / \mathrm{s}$ at any flow rate. Clearly, both guidelines seek to ensure that severe, not moderate, $\mathrm{AS}$ is present. Either contractile reserve or a high aortic velocity, 
with a persistently small valve area, offers convincing evidence that removing the valve obstruction will benefit the patient.

In symptomatic patients with low-flow, low-gradient severe AS with normal EF, the ACC/AHA guidelines recommend AVR when AS is the most likely cause of symptoms, but the ESC guidelines consider this only a Class IIa indication. Both guidelines emphasise the need to: (1) measure AS severity when the patient is normotensive, (2) ensure stroke volume index is $\leq 35 \mathrm{ml} / \mathrm{m}^{2}$, (3) index valve area for body size (particularly in smaller patients), and (4) consider computed tomographic quantitation of valve calcification. Other possible causes of symptoms should also be diagnosed and treated before concluding AS is the cause of symptoms.

\section{ASYMPTOMATIC SEVERE AS WITH LV DYSFUNCTION}

In addition to recommending AVR for asymptomatic adults with severe high-gradient AS and an LVEF $<50 \%$, AVR now is considered reasonable with an $\mathrm{EF}<55 \%{ }^{1}$ or a progressive decline to $<60 \%{ }^{2}$, based on advanced imaging modalities showing LV myocardial changes long before symptom onset ${ }^{3}$.

\section{ASYMPTOMATIC SEVERE AS WITH MARKERS OF RAPID SYMPTOM ONSET}

Both guidelines concur that AVR is reasonable in patients with severe AS at low surgical risk when rapid disease progression predicts an impending onset of symptoms. Markers of rapid progression include very severe AS (velocity $>5 \mathrm{~m} / \mathrm{s}$ ), a serum BNP level 3 -times normal, an increase in velocity $\geq 0.3 \mathrm{~m} / \mathrm{s} / \mathrm{yr}$ or severe valve calcification. Neither guideline recommends AVR for patients with asymptomatic AS who do not meet these criteria, although early small randomised trials with short-term follow-up are promising and several larger trials are in progress ${ }^{4,5}$.

\section{Choice of valve intervention}

The ESC/EACTS and ACC/AHA guidelines take slightly different approaches to thinking about the choice of intervention in patients with an indication for AVR, but the final recommendations are remarkably similar (Figure 1). Both guidelines emphasise the need for shared decision making with the patient in the context of a Heart Valve Team. Both guidelines recommend palliative care if

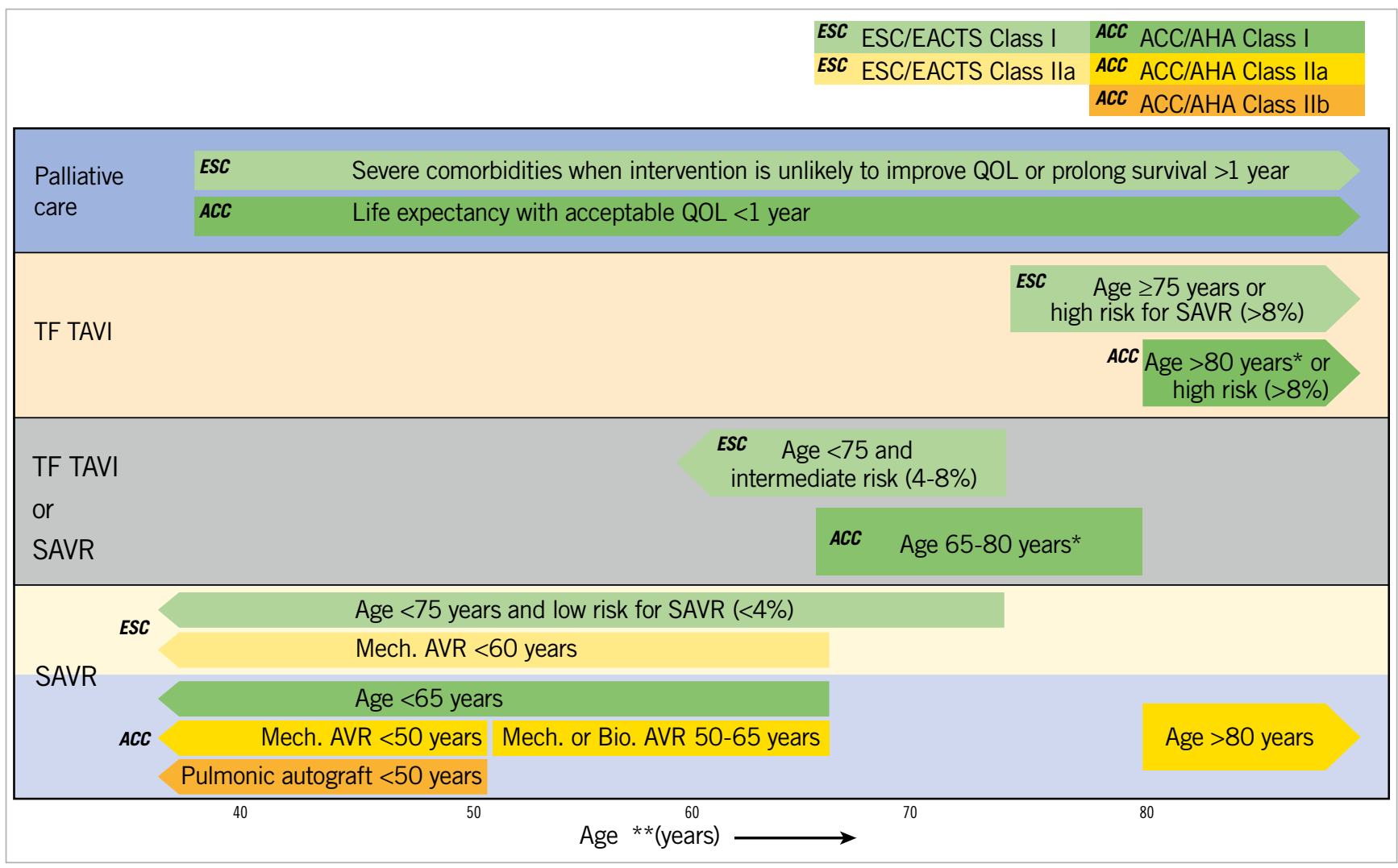

Figure 1. Choice of intervention in patients with severe aortic stenosis (AS) meeting guidelines for aortic valve replacement (AVR) in the ACC/AHA and ESC guidelines. Schematic showing major recommendations; additional details are included in each document ${ }^{1,2}$ Recommendations are only for transfemoral (TF) transcatheter aortic valve implantation (TAVI), not for other access routes. Estimated surgical risk is based on established scores plus additional considerations. The ESC recommendation for either TAVI or surgical AVR (SAVR) in patients under the age of 75 years with an intermediate surgical risk is truncated because these guidelines recommend a mechanical AVR under the age of 60 years. *ACC/AHA recommendations for TAVI only include patients with a Class I indication for AVR. surgical AVR $(S A V R)$ is recommended for Class IIa indications. **Age is used as a surrogate for expected remaining years of life with decision making individualised for each patient, also considering quality of life ( $Q O L)$. 
life expectancy with an acceptable quality of life is $<1$ year, even with intervention.

In both guidelines, the key concepts underlying the choice of transcatheter aortic valve implantation (TAVI) versus surgical AVR (SAVR) are estimated surgical risk and the balance between life expectancy and known valve durability ${ }^{6}$. TAVI is recommended if a transfemoral (TF) approach is possible when surgical risk is high. In addition, the known durability of TAVI valves, $>5$ years, supports TAVI as the preferred choice in older adults, regardless of surgical risk.

Although there is little data on TAVI valve durability beyond 5 years or in younger patients, most patients prefer the less invasive approach with a shorter hospital stay, less pain, and a more rapid return to normal activities. Thus, either TF TAVI or SAVR is recommended in patients aged 65 to 80 years $^{2}$ or under the age of 75 years $^{1}$. While ESC/EACTS limits this recommendation to patients with an intermediate surgical risk, the ACC/AHA recommendation includes patients both at low and intermediate risk.

SAVR remains recommended, in preference to TAVI in younger patients, particularly when surgical risk in low, given the known limited durability of bioprosthetic valves. A mechanical AVR is considered reasonable in patients under the age of 60-65 years based on a very low rate of repeat intervention which offsets the risk of lifelong warfarin anticoagulation. Conversely, a bioprosthetic SAVR is reasonable in older patients. The ACC/AHA guidelines also suggest that options that do not require a prosthetic aortic valve may be considered in selected patients at centres with experience in these approaches.

\section{Future directions}

The new ESC/EACTS and ACC/AHA guidelines for management of adults with valvular heart disease provide high-quality clinical guidance supported by a solid evidence base. It is expected that guidelines might differ regionally to account for specific patient populations, health care systems, social norms, economic issues, etc., as illustrated by the recent UK and South American guidelines $^{7,8}$. However, we all would benefit from collaboration to develop and maintain an evidence base for valvular heart disease $^{9}$. Expert (but volunteer) writing group members do not have the expertise or time needed to rigorously review and present the numerical data; instead, we need information specialists, statisticians, and data presentation experts. We need to involve a broader group of stakeholders in the guideline development process, including patients, policy makers, and non-specialist experts in clinical decision making. We must address racial/ethnic and gender disparities in diagnosis and management ${ }^{10}$. Finally, there is a need for continuous updates; even in the short time since development of the ESC/EACTS guideline, potentially practice-changing clinical studies have been published ${ }^{5}$. Going forward, we should work together to develop a shared evidence database that is rigorous, clearly presented and continuously updated. This common foundation can then be used by each professional society for guideline development.

\section{Conflict of interest statement}

The author has no significant financial conflicts of interest relevant to this topic.

\section{References}

1. Vahanian A, Beyersdorf F, Praz F, Milojevic M, Baldus S, Bauersachs J, Capodanno D, Conradi L, De Bonis M, De Paulis R, Delgado V, Freemantle N, Haugaa KH, Jeppsson A, Jüni P, Pierard L, Prendergast BD, Sadaba JR, Tribouilloy C, Wojakowski W. 2021 ESC/EACTS Guidelines for the management of valvular heart disease. EuroIntervention. 2022;17:e1126-96.

2. Otto CM, Nishimura RA, Bonow RO, Carabello BA, Erwin JP 3rd, Gentile F, Jneid H, Krieger EV, Mack M, McLeod C, O'Gara PT, Rigolin VH, Sundt TM 3rd, Thompson A, Toly C. 2020 ACC/AHA Guideline for the Management of Patients With Valvular Heart Disease: A Report of the American College of Cardiology/American Heart Association Joint Committee on Clinical Practice Guidelines. Circulation. 2021; 143:e72-227.

3. Calin A, Mateescu AD, Popescu AC, Bing R, Dweck MR, Popescu BA. Role of advanced left ventricular imaging in adults with aortic stenosis. Heart. 2020;106: 962-9.

4. Kang DH, Park SJ, Lee SA, Lee S, Kim DH, Kim HK, Yun SC, Hong GR, Song JM, Chung CH, Song JK, Lee JW, Park SW. Early Surgery or Conservative Care for Asymptomatic Aortic Stenosis. N Engl J Med. 2020;382:111-9.

5. Banovic M, Putnik S, Penicka M, Doros G, Deja MA, Kockova R, Kotrc M, Glaveckaite S, Gasparovic H, Pavlovic N, Velicki L, Salizzoni S, Wojakowski W, Van Camp G, Nikolic SD, Iung B, Bartunek J; AVATAR-trial investigators. Aortic Valve ReplAcemenT versus Conservative Treatment in Asymptomatic SeveRe Aortic Stenosis: The AVATAR Trial. Circulation. 2021 Nov 13. [Epub ahead of print].

6. Oyetunji SO, Otto CM. Transcatheter aortic valve implantation or replacement? Valve durability in the context of patient life expectancy. Eur Heart J. 2021;42: 2920-3.

7. Garbi M. National Institute for Health and Care Excellence clinical guidelines development principles and processes. Heart. $2021 \mathrm{Feb} 23$. [Epub ahead of print].

8. Lamelas P, Ragusa MA, Bagur R, Jaffer I, Ribeiro H, Baranchuk A, Wyss F, Sosa Liprandi A, Olivares G, Arrais M, Rendon JC, Catrip J, Agatiello C, Cura F, Marchena A, Sandoli de Brito F, Jr. , Mangione JA, Damonte A, Santaera O, Hidalgo P, Nieuwlaat R, Izcovich A; Endorsed by the Sociedad Latino Americana de Cardiologia Intervencionista (SOLACI) and the Sociedad Interamericana de Cardiología (SIAC). Clinical practice guideline for transcatheter versus surgical valve replacement in patients with severe aortic stenosis in Latin America. Heart. 2021;107:1450-7.

9. Otto CM, Kudenchuk PJ, Newby DE. Cardiovascular professional societies fall short in providing impartial, clear and evidence-based guidelines. Heart. 2021 Feb 26. [Epub ahead of print].

10. Lamprea-Montealegre JA, Oyetunji S, Bagur R, Otto CM. Valvular Heart Disease in Relation to Race and Ethnicity: JACC Focus Seminar 4/9. J Am Coll Cardiol. 2021; 78:2493-504 